Annales Geophysicae (2002) 20: 1469-1477 (C) European Geophysical Society 2002

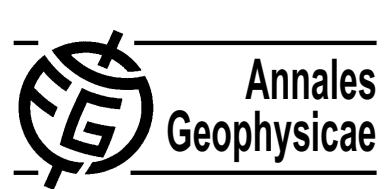

\title{
High resolution general purpose D-layer experiment for EISCAT incoherent scatter radars using selected set of random codes
}

\author{
T. Turunen, A. Westman, I. Häggström, and G. Wannberg \\ EISCAT Scientific Association, P.O.Box 164, SE-981 23 Kiruna, Sweden
}

Received: 5 November 2001 - Revised: 14 August 2002 - Accepted: 20 August 2002

\begin{abstract}
The ionospheric D-layer is a narrow bandwidth radar target often with a very small scattering cross section. The target autocorrelation function can be obtained by transmitting a series of relatively short coded pulses and computing the correlation between data obtained from different pulses. The spatial resolution should be as high as possible and the spatial side lobes of the codes used should be as small as possible. However, due to the short pulse repetition period (in the order of milliseconds) at any instant, the radar receives detectable scattered signals not only from the pulse illuminating the D-region but also from 3-5 ambiguous-range pulses, which makes it difficult to produce a reliable estimate near zero lag of the autocorrelation function. A new experimental solution to this measurement problem, using a selected set of 40-bit random codes with $4 \mu$ s elements giving $600 \mathrm{~m}$ spatial resolution is presented. The zero lag is approximated by dividing the pulse into two 20-bit codes and computing the correlation between those two pulses. The lowest altitudes of the E-layer are measured by dividing the pulse into 5 pieces of 8 bits, which allows for computation of 4 lags. In addition, coherent integration of data from four pulses is used for obtaining separately the autocorrelation function estimate for the lowest altitudes and in cases when the target contains structures with a long coherence time. Design details and responses of the experiment are given, and analysed test data are shown.
\end{abstract}

Key words. Radio science (signal processing); Ionosphere (plasma temperature and density; instruments and techniques)

\section{Introduction}

Incoherent scatter radars measure the D-layer by using the so-called pulse-to-pulse correlation method. The target autocorrelation function is estimated as a function of range

Correspondence to: T. Turunen

(tauno.turunen@eiscat.com) by correlating samples from several separate target illuminations. This arrangement must be used because the target coherence time below roughly $90 \mathrm{~km}$ altitude is longer or much longer than the longest possible pulse length, which can be used at such a close range. From the obtained target autocorrelation functions one can estimate the target cross section, which is proportional to the electron density. The Doppler shift gives bulk motion of the scattering media for any volume and finally, the target spectrum can be estimated. The spectral shape in the D-layer is theoretically Lorentzian and the measurements support this at least within the accuracies obtained so far. The spectral width depends on several parameters such as temperature, ion masses and presence of negative ions. The incoherent scatter measurements can be effectively used to study the D-region aeronomy. An extensive review on this has been given by Turunen (1986), where one can also find references to the related theoretical background.

The method described in this paper has been originally developed for EISCAT UHF (925 MHz) and EISCAT VHF $(224 \mathrm{MHz})$ incoherent scatter radars. D-layer measurements by incoherent scatter are very demanding because under normal conditions the target cross section is very low. For obtaining simultaneously both the required high spatial resolution and the efficient use of the radar duty cycle, one has to apply effective modulation methods. Since the nonzero extent of the wanted target autocorrelation function is of the order of milliseconds, one has to transmit pulses using as short as possible interpulse periods as the radar transmitter parameters. Interpulse periods down to 1.3-2 milliseconds can be used in practice in the EISCAT radars. The transmitted pulse length is always a compromise. It should be as long as possible for obtaining the best signal-to-noise ratio and as short as possible, to allow sampling at the lowest altitudes. Transmitted pulses of less than $200 \mu$ s in duration allow one, in practice, to start the target sampling from around $70 \mathrm{~km}$, which, under most circumstances, is about the lowest altitude where the D-layer becomes detectable. If the transmitted pulses are shorter than $100 \mu \mathrm{s}$, the transmitter duty 
cycle becomes used ineffectively in high duty cycle radars like EISCAT UHF and VHF radars, with a $12.5 \%$ transmitter duty cycle. If the experiment uses high modulation bandwidth for obtaining very high spatial resolution, then limitations in the real-time computations may become the factor dictating the maximum pulse length.

To obtain an estimate of the scattered signal power, one can measure an estimate for the target autocorrelation function in the vicinity of the zero lag, i.e. at delays which are less than the duration of the transmitted pulse. This is much more practical than measuring the true power, since the expectation value of the white noise contribution becomes nearly zero with a correctly selected impulse response function of the receiving system, and so one does not need to estimate the system noise level. Simultaneously, high cancellation of the returns from the 3-5 nearest earlier transmitted pulses is needed. Those pulses illuminate unwanted scattering targets having non-zero correlations at delays shorter than the pulse length. In a typical D-layer experiment, the antenna beam directions used are close to vertical, and so the nearest and strongest disturbing volumes are at about the 250 $300 \mathrm{~km}$ altitude, near the F-layer maximum when using the shortest practical pulse repetition periods for D-layer experiments in EISCAT radars. Disturbing volumes much above the $1000 \mathrm{~km}$ range are of no importance, due to the low signal level that results from low target cross section and large distance.

There are no practical possibilities to cause the radar's blind pulses to be transmitted at the same frequency, thereby illuminating disturbing scattering targets at relatively short distances. The best one can do is to try to make the expectation value of the disturbing signal as close to zero as possible in the process used in making an estimate for the wanted target. A simple way to do this is to use modulations with a property that cross-correlates between modulation patterns to form a zero mean process at all delays. Random modulation, where every pulse has different and totally independent modulation, is a solution that always works. However, because the process cannot contain infinitely many pulses, the rejection is not perfect, but can be arranged to be good enough.

At long lags computed by the pulse-to-pulse correlation method, the remote pulse rejection is not necessarily needed in a similar way, because the cluttering targets do not correlate at those delays and the expectation value of the clutter signal approaches zero. However, any possible remote instantaneous pulse rejection improves the statistical accuracy of the measurement, because the dominating disturbing noise is often due to scattering from unwanted illuminated targets in the radar beam.

If the target can be measured using a longer interpulse period, then the remote pulse clutter becomes smaller and if the interpulse period can be lengthened, such that the nearest cluttering volumes are well above the F-layer peak, very substantial benefits can be obtained, especially if the radar has large power-aperture product. At the Arecibo radar, the integration time needed for a given accuracy could be shortened by about two orders of magnitude in velocity measurements, by increasing the interpulse period from $1 \mathrm{~ms}$ to $4 \mathrm{~ms}$ in an experiment using 13-bit Barker coded pulses with a $600 \mathrm{~m}$ spatial resolution (Zhou, 2000). It is clear that one can obtain similar improvements when measuring power approximation using an autocorrelation function estimate measured near the zero lag. One can make the experiment even better by keeping the shortest possible interpulse period and multiplexing the experiment to several different frequencies, for example, four. However, when the task is to measure the autocorrelation function of the target at several lags for accurate spectral width measurements, then increasing the interpulse period rapidly lowers the highest altitude at which the target is correctly sampled.

At high latitudes in the summertime, radars often see almost coherent scatter from very thin and sometimes very strongly scattering layers called Polar Mesospheric Summer Echoes (PMSE). A good low altitude experiment should give reasonably good estimates for those layers as well, and a good experiment should not become distorted due to these strong localized targets.

Auroral zone D-layer ionisation is often caused by the high-energy component of the particle precipitation. The softer component of precipitation ionises the E-layer and lower F-layer altitudes. The ideal D-layer experiment should contain at least some kind of E-layer measurement too. The spatial resolution should be as high as possible. However, one always pays a price for this in the signal-to-noise ratio, and the computational demands increase rapidly when increasing the spatial resolution. Some compromise has to be made and in the solution described here, a spatial resolution of $600 \mathrm{~m}$ is used in the starting solution from which other solutions can be developed, if needed.

Extreme time resolutions of the order of one second or less cannot be obtained in radar measurements in D-layer experiments. The widest target bandwidth which can be safely sampled using a $2 \mathrm{~ms}$ interpulse period is necessarily less than $250 \mathrm{~Hz}$ and obtaining a high enough number of independent samples from so low a bandwidth target demands necessarily some tens of seconds or more, even when the measuring conditions are good. Also, the spatial resolution demand is so high that the possibilities to speed up the experiment by using spatial filtering are very limited.

PMSE usually give very high signal-to-noise ratio, but the target bandwidth is so narrow (only a few Hz or less) that obtaining statistically significant estimates is a very slow process.

The target cross section of the D- and E-layer target varies from levels where the target is undetectable to levels where the signal is totally dominating. The gradients in the target cross section can be extremely high. Order-of-magnitude changes in the cross section over a distance of the order of $1-2 \mathrm{~km}$ are possible, e.g. in the surrounding mesopause or in the bottom of the E-layer. The target may also contain very localised enhancements of the cross section, like sporadic E-layers and PMSE. This kind of target is difficult for any radar. Heavily modulated measuring solutions are needed, 
and those solutions usually produce unwanted spatial effects, i.e. spatial ambiguities. These spatial ambiguities, which are often called "side lobes", can be considered from different starting points. The important characteristics are their sizes, the distribution of signs, and the sum of the side lobes. It is always beneficial if all the individual side lobes are small, but in particular, if the number of spatial ambiguities is high, the sum of the spatial ambiguities becomes important.

There are some basic principles which simplify the search for empirical solutions needed in low altitude experiments. At practical incoherent scatter frequencies in all D-layer measurements and in most E-layer measurements, the target bandwidth is narrow or very narrow compared with the modulation bandwidth needed for the required spatial resolution. The simplest approach is to then utilize the long coherence time of the target by using binary phase-modulated transmissions, which are then decoded in the amplitude domain.

D-layer experiments can be arranged without measurement of the zero lag, i.e. "true power", of the target autocorrelation function. It is better to approximate the true power by measuring a value near the zero lag. One can then arrange the distribution of the spatial ambiguities and the remote pulse clutter contribution to approach a zero mean so closely in the process that the error becomes insignificant. Second, the impulse response function of the receiver can be matched so closely to the needed sampling interval that the expectation value of the white noise contribution vanishes. Thus, no background measurement and related background subtraction are needed. These two features simplify the experiment quite a bit. The task is now to find such modulations for the experiment which fulfill these requirements, so that all unwanted responses approach a level that is not disturbing.

In theory, it is not difficult to arrange all this by using true random binary phase codes. However, because one must perform the measurement in the EISCAT radars with a preprogrammed set of codes, one should try instead to obtain a good enough solution by using a carefully selected set of random codes. This solution is used here. There are also fully deterministic solutions, which are totally or almost totally free from any ambiguities. Those solutions exist separately for pulse-to-pulse correlation experiments, for a zero-lag approximation with perfect remote pulse clutter cancellation and for the E-layer measurements, but more work is needed for finding a practical deterministic solution that contains all the wanted properties simultaneously.

\section{Possible modulations for a low altitude experiment}

Random or pseudo random binary phase codes and a few different deterministic codes are among the possible modulations which can be considered, where the coding elements can be further phase coded, for example, by using Barker codes. Among the deterministic codes are also solutions based on so-called complementary codes, which are in EISCAT radars used in PMSE measurements ( $\mathrm{La} \mathrm{Hoz} \mathrm{et}$ al., 1989). Deterministic codes can also be pulse codes or al- ternating codes with phase coded modulation elements. Most D-layer experiments in EISCAT radars have been carried out so far by using a Barker coded two-pulse code with staggered pulse separations and phase inversion in the second pulse (Turunen, 1986), but better experiments can now be developed.

Among the most effective modulations created for incoherent scatter measurements are the alternating codes (Lehtinen and Häggström, 1987). Those codes have not been used so far in D-layer pulse-to-pulse correlation experiments. At least three different solutions exist for using them in D-layer experiments, but not all of these give the possibility for remote pulse cancellation, which is needed when trying to estimate the target $\mathrm{ACF}$ at delays near zero lag. By Barker coding the elements in alternating codes, one can keep the code lengths, the number of codes and the amount of computations at a manageable level, but then spatial ambiguities arise. Without using Barker codes as the lowest level of modulation, very long alternating codes are needed and the number of different code groups grows correspondingly. The known solutions will not be described here. The possible solutions based on alternating codes will be studied further.

A target can be correctly measured by using pulse-to-pulse correlation if, and only if, the target spectrum can be correctly sampled at the pulse repetition period. The length of the individual pulses used is, in practice, a little less than $10 \%$ of the pulse repetition period in EISCAT high duty cycle radars. A target that can be measured by pulse-to-pulse techniques is necessarily highly coherent on the time scale of the basic pulse length. The basic pulses can, therefore, without any limitation be phase modulated with long phase codes to form a single coded pulse. With suitably chosen codes one can then, for any wanted spatial resolution, obtain the lowest possible noise bandwidth, the simplest possible target illumination solution and a simple data structure. Relatively straightforward algorithms can be used in the pulse-to-pulse correlation computations, the zero lag approximation and the E-layer part of the experiment.

\section{The use of randomly phase coded pulses in low-altitude experiments}

The basic solution for random codes is very simple. One first defines the spatial resolution, which gives the length of the modulation element. For a $600 \mathrm{~m}$ resolution, it is $4 \mu \mathrm{s}$. The next step is to decide the total pulse length. If one wants to be safely ready to start receiving at about the $60 \mathrm{~km}$ range or below, then in the present EISCAT radars $160 \mu \mathrm{s}$ is a good total pulse length and with a $600 \mathrm{~m}$ spatial resolution, this leads to 40-bit code.

The key factor in the signal processing that makes our approach different from methods used previously is that for every range bin, the 40 information-carrying samples are processed in three different ways, i.e. they are matched-filter decoded by filters of three different lengths. 


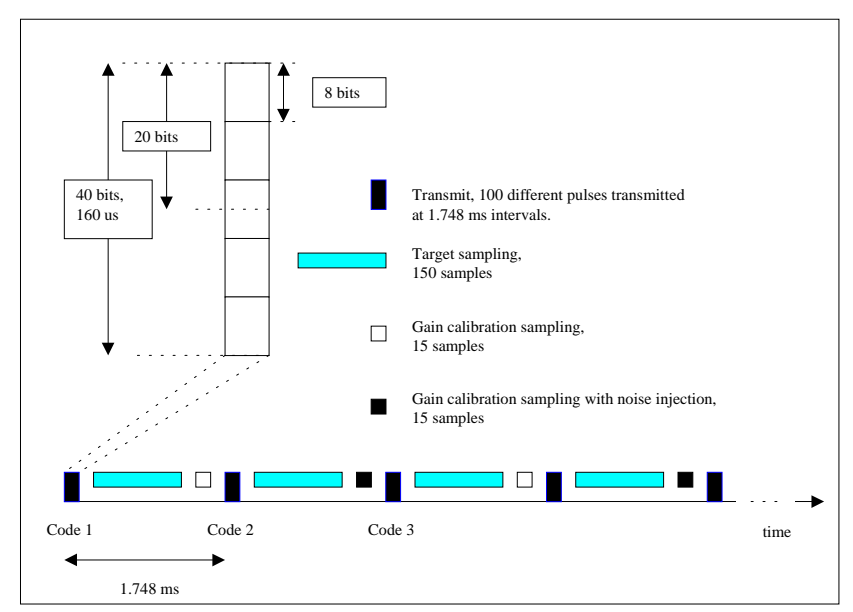

Fig. 1. Transmitted pulse structure and sampling arrangements.

The decoding using 40-bit FIR filtering produces data for pulse-to-pulse correlation computations, where the lag increment is the same as the pulse repetition period. In the next step, the sample vector is divided into two 20-bit parts, whose parts are decoded separately and from the outputs a series of $80 \mu$ s lags is computed as an approximation of the "true power". In this way, providing that the filters are behaving well, one avoids the need for background subtraction. Finally, the 40-bit sample vector is divided into 5 pieces of 8 bits, which are decoded separately. All the possible four lag profiles at $32 \mu \mathrm{s}$ lag increments are then computed to obtain E-layer data. Those profiles can also be used when estimating the accuracy of the zero lag approximation in the D-layer by checking against the gradient of the autocorrelation function near the zero lag.

The lag profile at the $80 \mu$ s delay is used here as an approximation of the power. Of course the result is not true power, i.e. the zero lag of the target autocorrelation function. It is rather a lag whose weighting function in the lag domain peaks at a $80 \mu$ s delay and whose weighting function covers the delays from 0 to $160 \mu \mathrm{s}$. The magnitude of the lag is, in most cases, a perfectly good approximation for the power of the scattered signal and in practice, it is often enough to use the real part of the lag as an approximation, because the imaginary part is always very small compared with the real part. The estimate has a non-zero imaginary part containing line-of-sight velocity information.

In the described method, all the unwanted responses, including remote pulse cancellation, form nearly zero mean processes if the codes are properly selected. In the EISCAT radars, one must use codes which are selected beforehand, and the code sequence is then transmitted repeatedly. A random code generator can generate those codes. However, most of the randomly generated individual codes are quite poor in terms of our criteria. Computing a large number of codes and selecting those that have good properties from the application point of view leads to a much better solution. In this work, less than one code out of $1 \cdot 10^{6}$ candidate codes

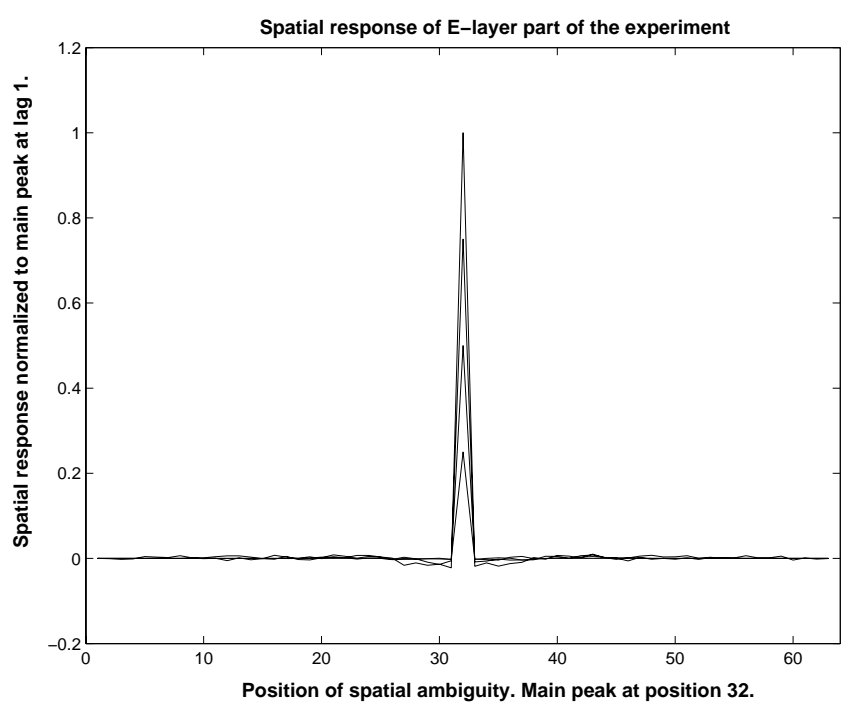

Fig. 2. Spatial response of the E-layer part of the experiment containing lag profiles at 32, 64, 96 and $128 \mu \mathrm{s}$.

passed all the tests.

It is not necessary to use boxcar weighted FIR filter coefficients in the decoding. By introducing a little tapering (fourth root of cosine is used in this work), one does not lose a lot of statistics, but it is easier to find suitable codes. In particular, it is possible to find codes with exceptionally small side lobes while simultaneously forcing the side lobes to have almost an exactly zero mean distribution. In the designed experiment, a similar window is used in every decoding (the whole 40-bit decoding, 20-bit decoding and 8-bit decoding). The window is only used for convenience, but it also gives certain benefits. Among other things it shapes the lag domain ambiguity functions to be slightly more peaked than a triangle.

The codes used in this experiment were selected in two phases. First, a few thousand codes were selected from a set of about $150 \cdot 10^{6}$ codes on the basis of their maximum side lobe amplitudes and the sum of the side lobes, simultaneously both for 40-baud and 20-baud compressions. The division to 8 bauds was not tested for side lobe performance. The codes were further studied by running them through all the processes, which have a tendency to create a non-zero bias. This is the zero lag approximation at $80 \mu$ s delay, as well as lags 1 and 2 computed from 8-bit division. Finally, 100 codes were selected from the remaining set in such a way that they produced close to zero mean distributions simultaneously in all unwanted responses. In this coding technique, one cannot totally remove all spatial ambiguities, but a totally acceptable level can be reached even with this low number of different codes. All transmitted pulses use totally independent codes, and this gives the remote pulse rejection. One could improve this a little by checking the transmitted order of the codes in a way that the clutter from the nearest disturbing pulse becomes minimized, but this was not done and evidently not needed.

The signal processing machinery limits the number of lags 
in the pulse-to-pulse correlation part of the experiment and in the programmed experiment, the computation was finally limited to 29 lags. At the lowest altitudes of the measured ranges, especially in the EISCAT VHF radar, and in the case of the Polar Mesospheric Summer Echos, one needs longer delays. For this purpose, a special "long coherence time mode" has been included in the experiment. Data from four pulses are added together in the amplitude domain to form 25 new data vectors from the original 100 vectors and pulse-topulse type correlation function estimates are then computed. The lag increment now becomes about $7 \mathrm{~ms}$, and 24 lags are computed in this mode. One should remember, however, that the lag domain ambiguity function contains altogether seven separate peaks.

Gain calibration, which is the only calibration needed, can be done using raw data, and one does not need to apply any decoding. Calibration data related to every other transmitted pulse contains a noise injection burst. After subtraction, one obtains the noise injection value, which is used to calibrate the gain. Both vectors contain a target signal, but it does not matter because the signal power is the same in both cases, although those two vectors do not have a single similar code in the transmission. In the designed experiment the real-time computations also include normalizations between different data types.

\section{Parameters and responses of the designed experiment}

It is practical to use a $4 \mu$ s baud length with the selected 40baud code length, resulting in a $600 \mathrm{~m}$ spatial resolution. The experiment uses a fixed set of 100 selected codes. The codes are given in Table 1, Appendix A as hexadecimal numbers. It is straightforward to derive the required 800 different FIR filters from the codes.

Sampling starts at the $60.0 \mathrm{~km}$ altitude. Altogether, 150 target data samples are taken, giving $66.6 \mathrm{~km}$ range coverage. This is followed by 15 samples for gain calibration. In every second radar cycle, these also contain noise injection. The total data vector after every transmitted pulse is thus 165 samples long. A 1.748 millisecond pulse repetition period is used, which gives a little over 9\% rf-duty cycle. Experimental arrangements are illustrated schematically in Fig. 1. One can find a more detailed formulation in Appendix A for some parts of the description given below.

The data from the complete set of 100 pulses is decoded using 8, 20 and 40 baud FIR filters compression. A window formed by using the fourth root of cosine function tapers the FIR filter coefficients used in the decoding. This window was used when searching for the codes, and it should be used for optimum performance with the given code set, although it is not absolutely necessary. Calibration power is computed without applying decoding. Finally, the lag profiles are computed and essentially three lag profile matrixes are formed.

In the computations, the data is normalised for obtaining convenient and safe values, e.g. from a dynamic range point of view. From the interpulse data one can compute lag pro-

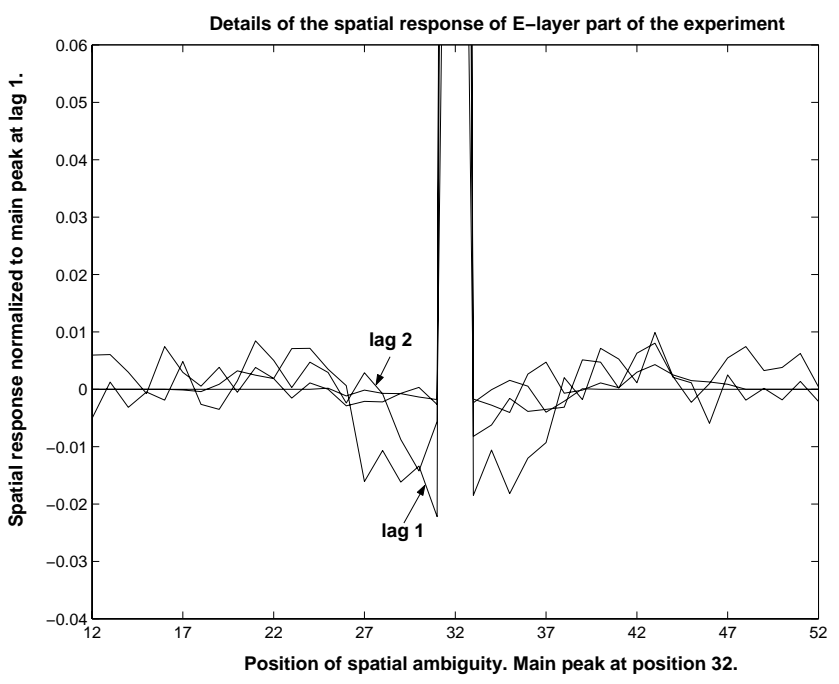

Fig. 3. Details of the spatial response of the E-layer part of the experiment.

files at 32, 64, 96 and $128 \mu$ s delays, using the output from the 8-bit decoding. These four lag profiles can be used as a simple E-layer data set, giving at least the Doppler shift and electron density with certain assumptions. The spatial resolution of $600 \mathrm{~m}$ also allows for, among other things, sporadic E-layer research. Although they do not form a good estimate for the autocorrelation function, these four lag profiles are much more informative than a mere power profile. They can also be used at D-layer altitudes. The spatial response of the code is shown in Figs. 2 and 3. The normalization is done so that the main lobe at lag 1 is set to 1 .

There is a tendency toward negative side lobes in lag 1 (32 $\mu$ s delay) and to a smaller extent also in lag 2 (64 $\mu$ s delay). This tendency is strongest near the main lobe and it is an unwanted effect. From practical experience it seems to be very difficult to suppress the shown behavior of spatial side lobes in lag profiles calculated from data using 8-bit compression when one must simultaneously keep the side lobe levels of 40- and 20-bit compressions low, and one has to keep the corresponding tendencies at the $80 \mu$ s lag profile computed from 20-bit compression at an acceptable level. A code selection was accepted when the worst case side lobes in the lag profiles computed from 8-bit compression were below $2 \%$ of the main lobe and the sum of the side lobes at any lag was below the 5\% level. Only a few near $2 \%$ side lobes exist with the large majority of side lobes falling well below the $1 \%$ level. On the other hand, the number of side lobes in this type of coding structure is very high, and if the side lobes do not have a close to zero mean, than the cumulative side lobe error can become severe. The sums of side lobes relative to the main peak for the given code set is from lag 1 to lag 4 as follows: $-1.2 \%,-2.5 \%,+2.0 \%$ and $-4.9 \%$. For comparison the cumulative side lobe error is at least $+7.1 \%$ in any experiment using a 13-bit Barker code as the lowest level modulation element. 


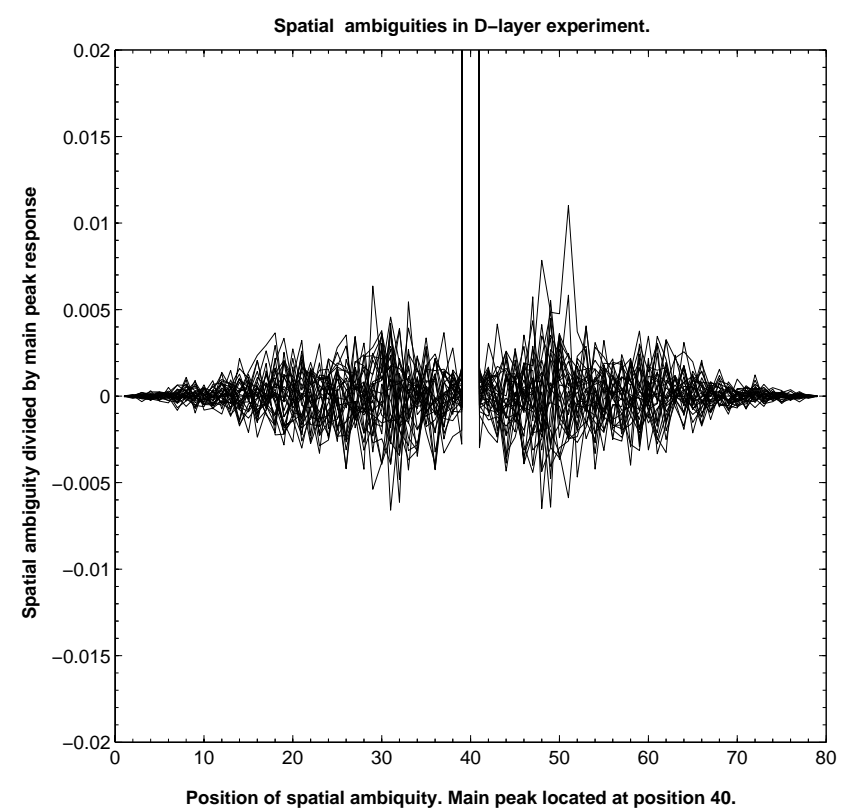

Fig. 4. Details of the spatial response of the D-layer part of the experiment. All the lags are superposed into the same picture. The main peak is normalized to one at every lag.

The side lobe structures of the $80 \mu \mathrm{s}$ lag and the pulse-topulse lags are shown superposed in detail in Fig. 4. At all lags the main lobes are normalized to unity. The envelope of the side lobes gives an indication of the worst case features. Only one side lobe, belonging to the $80 \mu$ s lag is near the $1 \%$ level. A majority of the side lobes are below the $0.5 \%$ level. Altogether, the number of side lobes in pulse-to-pulse computations is 79 and 39 in the $80 \mu$ s lag.

It is not easy to keep the sums of side lobes at a low enough level. The sums of side lobes relative to the main lobe are seen in Fig. 5. This error marginally exceeds the $4 \%$ level only in a single case, and in the majority of the cases, the error is below the $2 \%$ level. The side lobe sums are also behaving well in the sense that there are no clear systematic features. Since the side lobe structure is constant for any given set of codes, the side lobe correction can be done in the post processing of the data if the application demands it. Normally, it should not be necessary.

The lag profiles computed from coherently integrated data must be used only with a good understanding of the lag domain ambiguity function in such a method. In particular, users are warned about the first few lags, because there the target autocorrelation function can change a lot within the lag domain ambiguity function, which contains seven separate peaks. The data is useful mainly if the target has a very long coherence time such as at lowest altitudes, perhaps during the presence of negative ions and in connection with PMSE.

The performance of the codes used, shown in Table 1, was tested against 1000 totally random sets of codes using computations of 33 lags. The test parameters were as follows: The value of the worst side lobe, the sum of the absolute val-

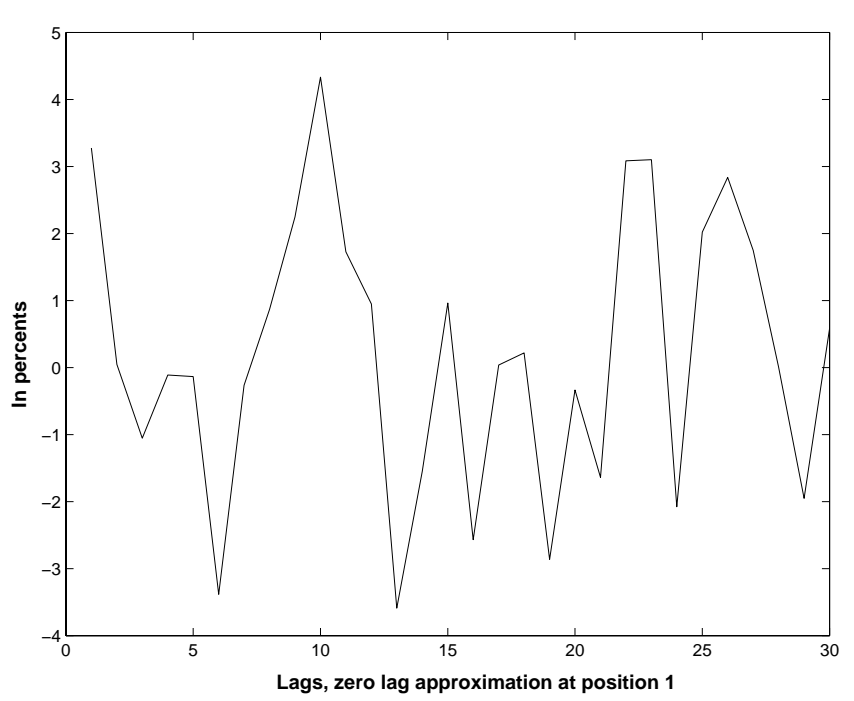

Fig. 5. Sums of the spatial ambiguities as a function of the lag in the D-layer part of the experiment, given in percents relative to the main peak.

ues of all side lobes and the number of side lobes exceeding the given thresholds. All computed lags were used in the comparison. The codes in Table 1 are better than any of the randomly selected codes used in all but one respect. It is easy to find a set of codes were the worst case side lobe is better than in the code set in Table 1. In fact, the mean value of the worst case side lobe in the randomly selected codes is about $2 \%$ lower than in the codes used, and the reason is that one of the side lobes (in the zero lag approximation) is much higher than the others, as seen in Fig. 4. On the other hand, as a mean, the number of side lobes with an absolute value greater than $0.3 \%$ of the main peak is almost twice as high in randomly selected codes, and if the threshold is increased to $0.4 \%$, more than three times as many side lobes exceed the threshold in the randomly selected codes. The sum of the absolute values of all the side lobes is as a mean $17 \%$ worse in randomly selected codes, and none of the test code sets was found to be better in this respect than the codes used in this work.

The coding could be improved by searching for a better set of codes, which is always possible but somewhat time consuming. One could also increase the number of codes from 100 up to the point allowed by the radar hardware.

The $600 \mathrm{~m}$ resolution code has been designed to be a general-purpose code and uses the radar resources well. There is also a simplified $150 \mathrm{~m}$ resolution version available, which can be used in special applications that demand very high spatial resolution.

Data from some of the first tests are shown in Fig. 6 for the D-layer target, from $70 \mathrm{~km}$ to about $100 \mathrm{~km}$ altitude. The basic parameters that one obtains from the D-layer are the power, spectral width and Doppler shift of the scattered signal. The curves are based on a least-squares fitting to all the available data, assuming a Lorentzian spectrum. Power is 


\section{EISCAT VHF 2001-06-21 0602:30-0605:00}
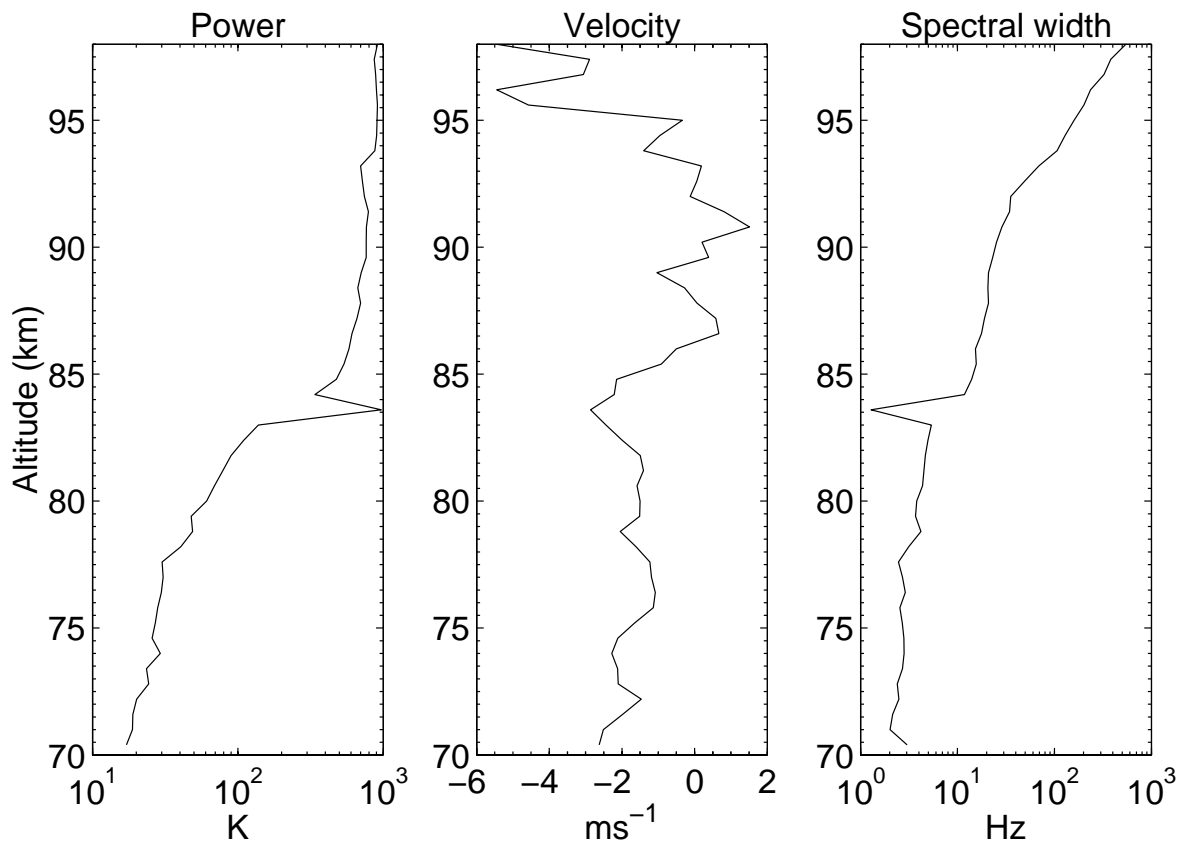

Fig. 6. Example data measured in Troms $\emptyset$ by EISCAT VHF radar at $224 \mathrm{MHz}$. Antenna pointing is vertical. The received power is expressed as a noise temperature in Kelvin, Doppler velocity in $\mathrm{ms}^{-1}$ and spectral width in Hz. Positive velocity means velocity is upwards. All three modes of lag profiles are used everywhere in the fitting and a Lorentzian shape of spectrum is assumed, which means that E-layer altitudes are not included in the analysis. Integration time is two and one-half minutes. Polar Mesospheric Summer Echo at $83.6 \mathrm{~km}$ altitude is seen in all measured parameters.

shown without range correction. Integration time is two and one-half minutes, and data containing satellite echo contamination have been rejected.

The target power varies by almost two orders of magnitude over the covered altitude range, and the spectral width changes by a factor of about 500 . The total Doppler velocity is less than $8 \mathrm{~ms}^{-1}$ over the altitude range. Positive velocity is upwards. A weak Polar Mesospheric Summer Echo is seen in the power data at $83.6 \mathrm{~km}$ altitude in one $600 \mathrm{~m}$ volume. It is also seen as a local deep spectral width minimum. The spectral width of the surrounding D-layer is significantly lower below the PMSE than above it. The altitude gradient of the Doppler velocity changes signs exactly at the PMSE altitude. In general the designed experiment seems to work well.

\section{Summary}

A new way to measure the low altitude ionosphere by incoherent scatter radar is described. Using a selected set of random 40-bit binary phase codes, the experiment produces D-layer pulse-to-pulse correlation lags and an approximation of power simultaneously, using a lag near the zero lag with high ambiguous range rejection. Lags are also computed for layers with a very long coherence time. Finally, a simple interpulse four-lag estimate is generated. In particular, this is intended for extending the measurement to the lower parts of the E-layer, but it covers all the measured ranges. A common modulation is used in all these modes.

Acknowledgement. EISCAT is an international association supported by Finland (SA), France (CNRS), the Federal republic of Germany (MPG), Japan (NIPR), Norway (NFR), Sweden (NFR), and the United Kingdom (PPARC).

Topical Editor M. Lester thanks C. Heinselman and another referee for their help in evaluating this paper.

\section{Appendix A The computations needed in the described D-layer experiment}

The experiment is schematically shown in Fig. 1. We denote the $i$ th modulation bit of the $m$ th transmitted pulse by $b_{m i}$. Any $b_{m i}$ can have a value +1 or -1 , indicating phase modulation by 0 or 180 degrees correspondingly. Transmission occurs in the order $i=1,2, \ldots, 40$. The experiment uses 100 different pulses with 40 bits modulation. After every transmitted pulse, the scattered signal from the target is sampled using a baud-matched sampling interval, i.e. identical with the bit length used in the modulation. We denote the complex samples taken after transmitting the $m$ th pulse by $x_{m j}$, where $j=1, \ldots, 150$. 
Table A1. Codes used in the designed experiment shown as hexadecimal numbers. Conversion to binary, including the possible leading zeros and replacing zeros with -1 , gives the 100 codes of 40 bits used. Codes are transmitted with the leftmost bit first. Every entry represents one 40-baud code. The first five codes are those forming the first row in the table

$\begin{array}{lllll}\text { A9471188B6 } & \text { DB9FC23E25 } & \text { 899EA7F359 } & \text { 4D62F2EBE6 } & \text { 5786483299 } \\ \text { 560674268E } & \text { FF1D8DD231 } & \text { DBF4393AE4 } & \text { FC74493AEF } & \text { 37D70ACFE1 } \\ \text { D4D16084D9 } & \text { 7914CD97FC } & \text { CE06A416B1 } & \text { CB23B4F1FC } & \text { C8A403ED15 } \\ \text { 3177EF26C6 } & \text { 539A222785 } & \text { 19E2D16443 } & \text { 0DAE5BCFAC } & \text { 32D7F49771 } \\ \text { 0B39FCC57F } & \text { E53B1B59F1 } & \text { A1FCDC5B8E } & \text { 8225EA5307 } & \text { 443448ADF2 } \\ \text { 054E181BBA } & \text { 26ABDDF28E } & \text { 97D9BB10FA } & \text { B31AA9F72E } & \text { 814C72E50D } \\ \text { 8B79933F56 } & \text { 0756301B8D } & \text { F4BBB2E519 } & \text { DEF0A959F4 } & \text { B1FCB8F266 } \\ \text { C194D38915 } & \text { 4C217E0959 } & \text { EC7AB8F929 } & \text { F75E6D2546 } & \text { 436AB3BBDA } \\ \text { B4E0A46159 } & \text { C20DDC1235 } & \text { 44E6F3EB3A } & \text { FCA937C5B4 } & \text { 329FDA9C9E } \\ \text { F20D4E120D } & \text { 9437DE2B7C } & \text { 36571ACFE9 } & \text { 4560E47A43 } & \text { 981A2AB623 } \\ \text { 5E985840D6 } & \text { EA47CBD9C3 } & \text { F7AE47CC2C } & \text { A62DFAF660 } & \text { ADB1CA75F2 } \\ \text { 68BBBC6E6 } & \text { 854C047DC5 } & \text { 70AC9046BA } & \text { BBC27E16EA } & \text { 3E4A60391A } \\ \text { 706BADDF1A } & \text { F7392EB92C } & \text { 2E7240A6B2 } & \text { 8BDF34E65C } & \text { 14C85CEC25 } \\ \text { 6A0A62A33C } & \text { B96FC519B6 } & \text { BB7AC8B3CA } & \text { A3781C096C } & \text { D0533A8171 } \\ \text { CE990A7103 } & \text { 32A01A61DF } & \text { 7282032BE7 } & \text { 9523841AF2 } & \text { 74E50113C5 } \\ \text { 6E104B7845 } & \text { 1D5BECA7D2 } & \text { 458D02F525 } & \text { 30A83AB4B1 } & \text { 7BA265C6FC } \\ \text { A99E31FBA6 } & \text { 30B95A5305 } & \text { CBC237F569 } & \text { F3A351EDE1 } & \text { 613ADA104D } \\ \text { 585E6FE93A } & \text { D389D8C085 } & \text { 4226E4B88B } & \text { B5C113C445 } & \text { 49C4243E71 } \\ \text { 2B21C451F8 } & \text { 5BD386CE7C } & \text { 2967C7B9F2 } & \text { C597EDD0E6 } & \text { E7D4D83D72 } \\ \text { C60645EC45 } & \text { A0B7351035 } & \text { 9503110FCF } & \text { EC0C29A0E5 } & \text { 6F0EB85FB1 }\end{array}$

For decoding the amplitude domain data, 8 different FIR filters are needed. For decoding the total pulse, the filter coefficients $c t_{m j}$ are the modulation bits of the pulse $m$ in reverse order

$c t_{m k}=b_{m(40+1-j)}, j=1,2, \ldots 40$.

The decoded data vector $z_{m t}$ for pulse $m$ is now

$z_{m t}=\sum_{j=1}^{40} x_{m(l-1+j)} * c t_{m j} * W_{j}$,

where $W_{j}$ is a weighting function. If the weighting function used $W_{j}=1$ for every $j$, then (2) represents the well-known matched filtering of phase coded data used extensively in radars, e.g. in connection with Barker codes or complementary codes. Our $W_{j}$ is chosen as a fourth root of cosine and this causes a small tapering of the filter coefficients:

$W_{j}=\sqrt[4]{\cos (\pi(j / k-(1+1 / k) / 2))}, k=40$.

The sample $z_{m t}$ contains the main target contribution from a single volume $V_{t}$. The spatial resolution along the radar antenna beam is given by the bit length in the modulation. In addition, the sample contains smaller contributions from many other volumes. If $W_{j}=1$ for every $j$, then the spatial response would be given by the autocorrelation function of the code used. The $W_{j}$ used modifies this a little. The wanted volume is obtained at zero lag of this autocorrelation function, and the other lags represent the unwanted contributions, namely the spatial ambiguities.

The modulations used are random. As a consequence, all the spatial ambiguities form a random zero mean process, and the spatial ambiguities of different codes do not correlate.

The target autocorrelation function estimate can now be computed for lags greater or equal to 1 . These are the pulseto-pulse correlation lags. For volume $t$ at lag $l$, the estimate computed from one set of $M$ codes is

$a c f_{t l}=\sum_{m=1}^{M-l} z_{m t} * z_{(m+l) t}^{*}$,

$M=100$ in the present solution.

This is done for every volume and all wanted lags and further time are averaged by repeating the process and accumulating like results. The lag increment is equal to the interpulse period. All contributions from unwanted volumes form a zero mean random process.

The next step is to estimate the power of the scattered signal for every volume, i.e. the zero lag of the target autocorrelation function. Simply squaring the samples given by Eq. (A2) does not work, because one cannot separate the contribution of white noise, power arising from spatial ambiguities and power arising from a few earlier transmitted pulses, which, in spite of having different modulations, increase the noise level. This problem is circumvented by approximating the power by the value of the autocorrelation function close to the zero lag instead of exactly at zero lag, because then one can make use of the good properties of the phase coding used. This method in a different arrangement has also been used earlier in EISCAT radars (Turunen, 1986). One of the possible ways is to treat the transmitted 40-baud pulse as a two-pulse, $2 \times 20$ baud code and compute the lag, which is allowed by this code. 
The following two filters having 20 coefficients are now formed for the transmitted pulse $m$.

$c A_{m p}=b_{m(40+1-j)}, j=1,2, \ldots, 20$,

$c B_{m q}=b_{m(20+1-j)}, j=1,2, \ldots, 20$.

Coefficients $c A_{m p}$ decode the target signal created by the first half of the transmitted pulse and the coefficients $c B_{m q}$ decode the contribution of the second half of the transmitted pulse. Two decoded data vectors are now formed for every pulse $m$

$x_{m t}=\sum_{j=1}^{20} x_{m(t-1+j)} * c A_{m p} * W_{j}, t=1,2 \ldots$,

$y_{m t}=\sum_{j=1}^{20} x_{m(t+20-1+j)} * c B_{m q} * W_{j}$.

The weighting function $W_{j}$ is given by formula (3), setting $k=20$. The filtered samples $x_{m t}$ and $y_{m t}$ represent the same volume $t$. The wanted zero lag approximation for volume $t$ is now

$a c f_{t 0}=\sum_{m=1}^{M} x_{m t} * y_{m t}^{*}$,

$M=100$ in the present solution.

This process is then time averaged for obtaining the required accuracy.

A simple "E-layer estimate" is also formed, which can be used to extend the range coverage above the altitude, where pulse-to-pulse correlation stops working. The computations are, in principle, identical to the previous zero lag approximation, where the transmitted pulse was treated as a two-pulse code, but now the pulse is treated as a five-pulse, $5 \times 8$ bauds code without any gaps between the pulses. Here the ambiguities disappear, because the basic code has been selected such that every subpulse has a different code. When decoded in the amplitude domain, and if the codes are truly random, the unwanted spatial responses form a zero mean random process. Altogether, 4 lags can be computed. The details of the formulation are not repeated here.

Finally, the experiment contains a special mode for targets with very long coherence time, e.g. PMSE. Coherently adding data of the type shown in Eq. (2) from 4 transmitted pulses produces 25 new data vectors, where the value $Z_{M t}$ for a volume $t$ is given

$Z_{M t}=\sum_{m=1}^{m=4} z_{(4(M-1)+m) t}, M=1,2, \ldots, 25$.

Finally, the pulse-to-pulse correlation type autocorrelation function estimate $A C F_{L t}$ is computed for lag $L$ and volume $t$

$A C F_{L t}=\sum_{M=1}^{25-L} Z_{M t} * Z_{(M+L) t}^{*}, L=1,2, \ldots, 24$.

The response of $Z_{M t}$ in time domain contains four narrow peaks separated by an interpulse period. As a consequence, the resulting lag domain ambiguity function for the estimates $A C F_{L t}$ has seven peaks separated by one interpulse period and the relative weights are 1,2, 3, 4, 3, 2 and 1. The strongest peak is centered at a nominal delay, which is a multiple of $4 L$ interpulse periods. One has to keep this somewhat poorly behaving time domain ambiguity function in mind when using the data.

\section{References}

La Hoz, C., M. Rietveld, G. Wannberg, and S.J Franke, The status and planned development of EISCAT in mesosphere and Dregion experiments, Handbook for MAP., 28, 476-488, 1989.

Lehtinen, M. S. and Häggström, I.: A new modulation principle for incoherent scatter measurements, Radio Sci., 22, 625-634, 1987.

Turunen, E.: Incoherent scatter radar contribution to high latitude D-region aeronmy, J. Atmos. Terr. Phys., 58, 707-752, 1996.

Turunen, T.: GEN-SYSTEM - a new experimental philosophy, J. Atmos. Terr. Phys., 48, 777-785, 1986.

Zhou, Qihou H.: Incoherent scatter radar measurement of vertical winds in the mesosphere, Geophys. Res. Lett., 27, 1803-1806, 2000. 